\title{
Bufalin Inhibits Cellular Proliferation and Cancer Stem Cell-Like Phenotypes via Upregulation of MiR-203 in Glioma
}

\author{
Tao Liu Chanji Wu ${ }^{a}$ Guohu Weng ${ }^{a}$ Zhongyan Zhao ${ }^{a}$ Xiangying He \\ Chuanyi Fu $^{\mathrm{b}}$ Zhiyan Suia Shixiong Huang ${ }^{\mathrm{a}}$

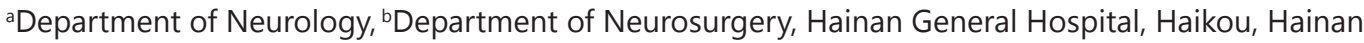 \\ Province, China
}

\section{Key Words}

Bufalin • MicroRNA-203 • Glioma • Cancer stem-like cell

\begin{abstract}
Background/Aims: Prior studies have shown that bufalin inhibits cellular proliferation and induces apoptosis in various human cancers. MicroRNA-203 (miR-203) has been shown to function as an important regulator of tumor progression at various stages. In this study, we investigated the effect of miR-203 expression and bufalin treatment on glioma cell proliferation and stem cell-like phenotypes. Methods: We used cell viability assay, colony formation assay, cell apoptosis assay and neurosphere formation assay to dectect the treatment effect of bufalin on U251 and U87 cells. Cells were transfected with the miR-203 mimic without bufalin treatment or cells were transfected with anti-miR-203 under bufalin treatment, the above expreiments were repeated. RT-PCR was employed to quantify miR-203 expression. Western blot was performed to detect the stem cell-like (CSC) markers, OCT4 and SOX2. Luciferase activity assay was used to determine whether the SPARC is the target of miR-203. Results: Bufalin treatment inhibited cell proliferation, colony formation, and CSC phenotypes and increased cell apoptosis and expression of miR-203. Furthermore, overexpression of miR203 led to similar outcomes as bufalin treatment with respect to the cell viability, colony formation, cell apoptosis and the phenotypes of glioma cells. While anti-miR-203 attenuated the inhibitory effects of bufalin as promoting cell proliferation, colony formation and CSC phenotyes and inhibiting cell apoptosis. In addition, we identified SPARC as a novel target gene of miR-203. Conclusions: These findings suggest that miR-203 plays an important role in bufalin's ability to inhibit the growth of glioma cells and the development of stem cell-like phenotypes.

(c) 2017 The Author(s)

Published by S. Karger AG, Basel
\end{abstract}

\section{Introduction}

Gliomas are among the most common aggressive primary brain tumors and account for the majority of cancers of the central nervous system. According to the WHO classification, 
glioma can be divided into four grades, with the 5-year survival rate of high grade gliomas less than 5\% (a median survival time of 12 months from diagnosis) [1]. Prognosis is poor and recurrence is high because malignant gliomas show low sensitivity to radiotherapy and chemotherapy [2]. Thus, the development of effective therapeutic strategies for glioma treatment is needed.

Bufalin is a cardiotonic steroid isolated from the traditional Chinese medicine ChanSu, which is dried toad venom or dried secretions from the skin glands of Bufo bufo gargarizans Cantor or Bufo melanostictus Schneider [3]. ChanSu has been used as a traditional Chinese medicine for thousands of years and has long been used to treat cancer [4]. In the past two decades, bufalin, the primary active ingredient of Chansu, has been shown to have high potential as an anti-tumor chemotherapeutic agent $[3,5]$, with antitumor effects reported in various cancers, including lung, gastric, breast, prostate, ovarian, and bladder cancer and leukemia [6-10]. However, bufalin's anti-tumor effects and especially its molecular mechanism in glioma have yet to be determined.

MicroRNAs (miRNAs) are endogenous small non-coding RNAs (19-25 molecules) that recognize their target sites by directly binding to the 3' untranslated region (UTR) of target mRNAs, resulting in mRNA degradation or repression of translation [11]. Accumulating evidence has revealed that miRNAs are crucial to brain physiology and tumorigenesis, especially in gliomas, where abnormally elevated levels of miR-10b, miR-21, and miR-93 and abnormally reduced levels of miR-101, miR-491, and miR-203 have been identified [12-14].

The expression of miR-203 was reported to decrease with ascending WHO tumor grade [15]. Recent studies have shown that miR-203 acts as a tumor-suppressive microRNA by targeting certain oncogenes involved in glioma cell proliferation, invasion, and drug resistance. For example, downregulation of miR-203 is associated with chemoresistance in human glioblastoma through the promotion of epithelial-mesenchymal transition (EMT) via SNAI2 [16]; overexpression of miR-203 drastically suppresses the protein expression of PLD2, inhibiting the proliferation and invasion of glioma cells [17]; and miR-203 was also demonstrated to sensitize glioma cells to temozolomide and inhibit glioma cell invasion by targeting E2F3 [18]. These findings clearly indicate that miR-203 plays a major role in the suppression of glioma tumor cell migration and invasion.

Given the likelihood of miR-203 and BF as novel glioma treatment candidates, we attempted to identify a relationship between miR-203 and bufalin treatment in glioma. We examined the effect of bufalin treatment on cell proliferation, colony formation, cancer stem cell-like (CSC) phenotype, and miR-203 level.

\section{Materials and Methods}

\section{Cell lines}

Human glioma cell lines U251 and U87 were obtained from the American Type Culture Collection (ATCC, Manassas, VA, USA). Cells were cultured in Dulbecco's modified Eagle's medium (DMEM) (HyClone, Logan City, UT, USA) supplemented with 10\% fetal bovine serum (FBS) (Gibco, USA), $100 \mathrm{U} / \mathrm{ml}$ penicillin and $100 \mu \mathrm{g} / \mathrm{ml}$ streptomycin (Sigma, St. Louis, MO, USA) in a humidified atmosphere containing $5 \% \mathrm{CO}_{2}$ at $37^{\circ} \mathrm{C}$.

\section{MicroRNA Expression}

Total RNA was isolated from U251 using TRIzol (Invitrogen, Grand Island, NY, USA) according to the manufacturer's protocol. The miRNA sequence-specific RT-PCR primers and endogenous control RNU6 were purchased from Qiagen (Hilden, Germany). The primer sequences are listed in Table 2. The relative expression was calculated by normalizing to that of RNU6.

Transfection of miR-203 mimic or inhibitor

Oligonucleotide has-miR-203 mimics (miR-203) were designed as described previously [19], and a non-specific sequence was used as a negative control (miR-NC). The miR-203 plasmid construct was 


\section{Cellular Physiology Cell Physiol Biochem 2017;44:671-681

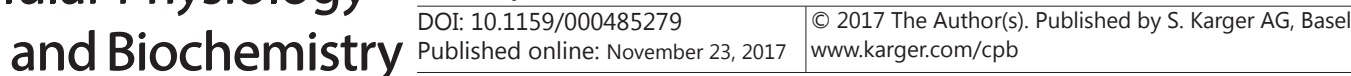

Liu et al.: Bufalin Inhibits Cellular Proliferation via MiR-203

Table 1. The sequence of miR-203 mimics and miR-NC miR-203-mimics 5'-GUGAAAUGUUUAGGACCACUAG-3'

5'-AGUGGUCCUAAACAUUUCACUU-3'

miR-NC
Table 2. The primers of miR-203 and RUN 6

miR-203 5'-GTCGTACCAGTGCAGGGTCCGAGGTATTCGCACTGGATACGACCTAGT-3'

5'-GCCCGTGAAATGTTTAGGACCAC-3'

miR-NC $\quad 5^{\prime}$-CTCGCTTCGGCAGCACA-3'

5'-AACGCTTCACGAATTTGCGT-3'

5'-ACGUGACACGUUCGGAGAATT-3'

chemically synthesized by Gene Pharma (Shanghai, China) and is shown in Table 1. The miR-203 inhibitor (anti-miR-203) was purchased from Qiagen, and scrambled control RNA (SCR) was used as the miRNA inhibitor negative control. U251 and U87 cells were seeded in a 6-well plate at $1 \times 10^{5}$ cells per well and cultured for $24 \mathrm{~h}$. Lipofectamine 2000 (Invitrogen) was used to transfect cells with the miR-203 mimic, miR-NC, anti-miR-203, or SCR inhibitor at a final concentration of $200 \mathrm{nM}$, according to the manufacturer's instructions. Medium was replaced with fresh media $24 \mathrm{~h}$ after transfection.

\section{Cell viability assay}

U251 and U87 cells were seeded in 96-well plates at a density of $2 \times 10^{4}$ cells $/ \mathrm{ml}$ in triplicate and then incubated overnight at different concentrations of bufalin or $0.1 \%$ DMSO (solvent control) in $100 \mu \mathrm{l}$ of DMEM medium for 24 or $48 \mathrm{~h}$. Cells were also transfected with the miR-203 plasmid construct, the empty vector, anti-miR-203, or SCR siRNA and incubated for $24 \mathrm{~h}$. After treatment, the cells were incubated in 200 $\mu \mathrm{l}$ DMEM/FBS containing $0.5 \mathrm{mg} / \mathrm{mL}$ MTT (Sigma). The supernatant was then discarded, and the cells were lysed in $200 \mu \mathrm{l}$ DMSO for $10 \mathrm{~min}$. Cell viability was evaluated by measuring optical density (OD) values at $490 \mathrm{~nm}$ on a SpectraMax 190 (Molecular Devices Sunnyvale, CA, USA).

\section{Colony formation assay}

The cells were resuspended in DMEM/F12 medium, and $5 \times 10^{6}$ cells were seeded into 6-well plates. After two days, the cells were treated with bufalin or transfected with miR-203 construct, and the media were replaced with fresh media. On the fifth day, cells were fixed with methanol and stained with crystal violate. The cell colony-forming units ( $>50$ cells) were manually counted in four random wells by two independent, blinded investigators.

\section{Detection of cell apoptosis}

Initially, $5 \times 10^{5} \mathrm{U} 251$ and U87 cells were seeded in $60 \mathrm{~mm}$ dishes and allowed to grow for $24 \mathrm{~h}$, followed by treatment with bufalin or transfection with miR-203 plasmid construct or miR-203 inhibitor. Cells were harvested with trypsin-EDTA, fixed with ice-cold 70\% ethanol, and incubated with $1 \mathrm{mg} / \mathrm{ml}$ RNase A solution (Sigma). Furthermore, cells were collected by centrifugation and stained with Annexin V/ PI (Annexin V-FITC/Propidium Iodide, eBioscience, San Diego, CA, USA). Apoptosis of EPCs was assessed by flow cytometry (Dakocytomation, Beckman Coulter, MA, USA).

\section{Neurosphere formation assay}

The human glioma stem cell-like cells, derived from glioma cell lines U87 and U251, were enriched using the serum-free clone formation method with 2\% B27 Neuro Mix (Invitrogen), $20 \mathrm{ng} / \mathrm{mL}$ epidermal growth factor (EGF), and $10 \mathrm{ng} / \mathrm{mL}$ basic fibroblast growth factor (bFGF) (PeproTech, Rocky Hill, NJ, USA). Then, the cells were treated with bufalin or transfected with miR-203 plasmid vector. Glioma stem celllike cells were plated at 1000 cells per well in 24-well plates. After culture for two weeks, the number of neurospheres that contained more than 20 cells was visualized and calculated under a microscope.

\section{Luciferase activity assay}

The 3 '-UTR of SPARC with a putative miR-203 targeting site (CAUUUCA) was cloned into the pmirGLO Dual-Luciferase miRNA target expression vector (Promega, Madison, WI, USA). Similarly, a SPARC-UTR-MUT 
Liu et al.: Bufalin Inhibits Cellular Proliferation via MiR-203

luciferase reporter plasmid with a site-directed mutated targeting site was used as a control. Cells were grown to 70\% confluence and co-transfected with miR-203 plasmid vector and SPARC-UTR-WT or SPARCUTR-MUT constructs using Lipofectamine 2000, while another group of cells was transfected with SPARCUTR-WT or SPARC-UTR-MUT luciferase reporter plasmids with or without bufalin treatment. The luciferase assay was performed using a dual luciferase reporter assay kit (Promega) according to the manufacturer's instruction.

\section{Western blotting}

Cells were lysed with PhosphoSafe ${ }^{\mathrm{TM}}$ extraction reagent (Merck Millipore, Darmstadt, Germany) containing $1 \times$ proteasome inhibitor (Sigma). Thirty micrograms of protein was loaded onto $10 \%$ gradient NuPAGE gels (Novex, San Diego, CA, USA) for electrophoresis and subsequently transferred onto polyvinylidene difluoride (PVDF) membranes (Millipore). Western blotting was performed using the primary antibodies glyceraldehyde 3-phosphate dehydrogenase (GAPDH) polyclonal antibody (1:5000 dilution in 5\% milk, Proteintech), SPARC monoclonal antibody (1:500, Abnova, Taibei), SOX2 polyclonal antibody (Abnova), and OCT4 polyclonal antibody (Proteintech) (1:1000) and secondary goat anti-rabbit antibodies (1:4000, PerkinElmer, USA). Band intensities were analyzed using Quantity One 4.6.6 software (Bio-Rad Laboratories, Inc., Hercules, CA, USA).

Statistical analysis

Data analysis was performed using SPSS 19.0 software (IBM SPSS, Armonk, NY, USA). All data are presented as the mean \pm SD. Statistical comparisons were performed using the Student's $t$-test or one-way analysis variance models. $\mathrm{P}<0.05$ was considered statistically significant.

\section{Results}

Bufalin represses glioma cell proliferation and stem cell-like phenotypes

Uncontrolled proliferation is one of the key features of cancer cells. To investigate the role of miR-203 in glioma, cell growth was examined by MTT assays, and cell viability (\% of control) was calculated. A significant gradual decrease in cell viability of both U251 and U87 cells was observed with increasing concentrations of bufalin $(0,5,10,20,40,80$, $200 \mathrm{~nm}$ ) (Fig. 1A). Thus, we chose $20 \mathrm{~nm}$ as the bufalin concentration used in subsequent experiments. The cell apoptosis rate was significantly higher in cells treated with $20 \mathrm{~nm}$ of bufalin than in untreated cells $(P<0.05)$ (Fig. 1B). The colony-forming capacity of cells was determined using the colony formation assay. Compared with control cells, reduced colony formation was observed in cells treated with bufalin $(P<0.05)$ (Fig. 1C). After culture for two weeks, the number of neurospheres that contained more than 20 cells was calculated. Reduced neurosphere formation was observed in cells treated with bufalin than in control cells $(P<0.05)$ (Fig. 1D). Finally, Western blot was used to determine relative levels of two important stem cell-like markers, OCT4 and SOX2. Bufalin-treated cells expressed lower levels of both OCT4 and SOX2 than control cells $(P<0.05)$ (Fig. 1E). Collectively, the findings suggest that bufalin represses the proliferation of glioma cells and the development of stem cell-like phenotypes.

\section{Bufalin increases the expression of miR-203}

Since miR-203 plays a major role in glioma cell migration and invasion, we wanted to determine whether a relationship between bufalin administration and miR-203 expression exists. We used RT-PCR to determine expression levels of miR-203. In U251 cells treated with $20 \mathrm{nM}$ of bufalin at $6,12,24$, and 48 hours, miR-203 expression gradually increased over time (Fig. 2A). To further explore the effect of bufalin on miR-203 expression, U251 cells were treated with increasing concentrations of bufalin $(0,5,10,20,40 \mathrm{nM})$. Relative miR-203 expression in U251 cells at 24 hours following treatment with $10 \mathrm{nM}$ of bufalin was significantly higher than in U251 cells treated with lower concentrations $(P<0.05)$. Furthermore, miR-203 expression gradually increased with increasing concentrations of bufalin treatment (Fig. 2B). Taken together, these results suggest that bufalin can directly induce the expression of miR-203. 
Fig. 1. Bufalin suppresses cell viability and stem cell-like phenotypes in U251 and U87 cells. (A) Cytotoxicity of different doses of bufalin on U251 and U87 cells at 24 and 48 hours. (BD) Cell apoptosis (B), colony formation (C) and neurospheres formed (D) were determined in U251 and U87 cells treated with $20 \mu \mathrm{M}$ for 24 hours. (E) Immunoblot analysis of OCT4 and SOX2 in U251 and U87 cells treated with $20 \mu \mathrm{M}$ for 24 hours.

MiR-203 expression emulates the effects of bufalin administration on glioma cells The miR-203 plasmid vector was transfected into U87 and U251 cells. MTT assays demonstrated that miR-203 transfection inhibited cell growth $(P<0.05)$ (Fig. 3A). Furthermore, flow cytometry demonstrated that cells treated with miR-203 had significantly higher rates of apoptosis than cells treated with miR-NC $(P<0.05)$ (Fig. 3B). Colony formation assays showed lower rates of colony formation in cells treated with miR-203 than in cells treated with miR-NC $(P<0.05)$ (Fig. 3C). The neurosphere formation assay also showed lower rates of neurosphere formation in the miR-203 group compared to the miRNC group $(P<0.05)$ (Fig. 3D). Finally, cells transfected with miR-203 expressed lower levels of both OCT4 and SOX2 $(P<0.05)$ (Fig. $3 \mathrm{E})$. These results suggest that miR-203 has the same inhibitory effects as bufalin in glioma cells.

Anti-miR-203 attenuates bufalinmediated inhibition of cell proliferation and stem cell-like phenotypes

To further explore bufalin's inhibitory effects on cell growth and repression of stem cell-like phenotypes, miR-203, anti-miR-203, and scrambled control miRNA inhibitor as a negative control were transfected into U251 and U87 cells. Cell growth was significantly increased following the transfection of anti-miR-203 $(P<0.05)$ (Fig. 4A). We then used bufalin to treat those cells transfected with antimiR-203 or SCR. Anti-miR-203 cells treated with bufalin had a significantly lower rate of apoptosis than SCR cells treated with bufalin $(P<0.05) \quad$ (Fig. 4B). Meanwhile, despite bufalin treatment, colony formation

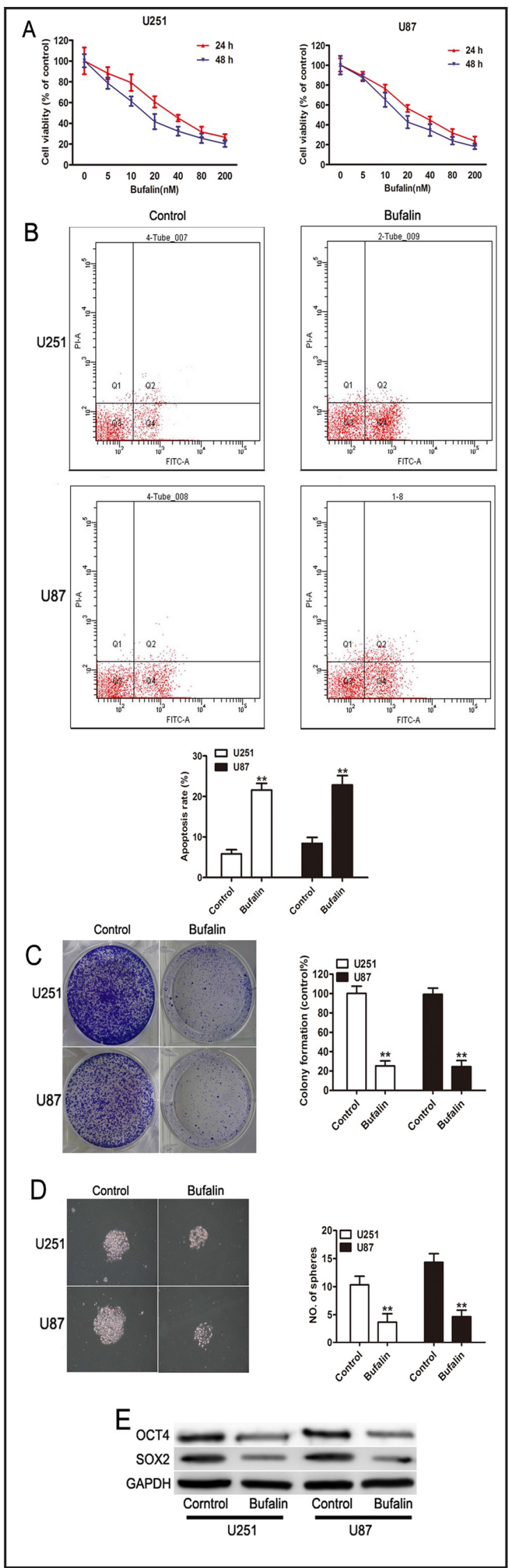


A

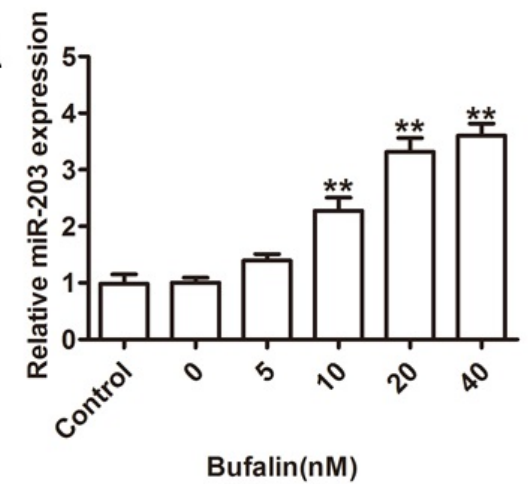

B

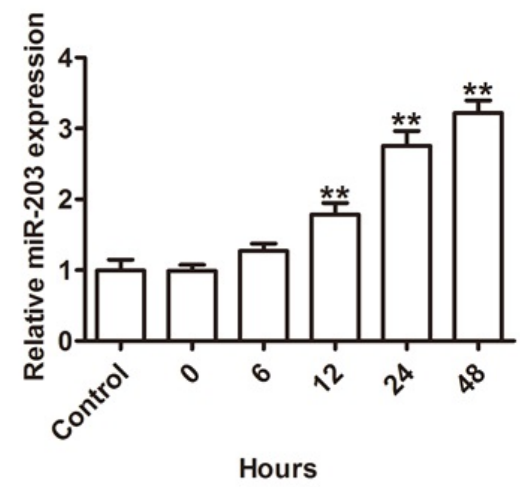

Fig. 2. Bufalin modulates miR-203 expression in human glioma cells. (A) miR-203 expression was analyzed in U251 cells treated with $20 \mu \mathrm{M}$ of bufalin at 6, 12, 24, and 48 hours. (B) Effects of bufalin on the expression of miR-203 at 24 hours following treatment with the indicated concentration.

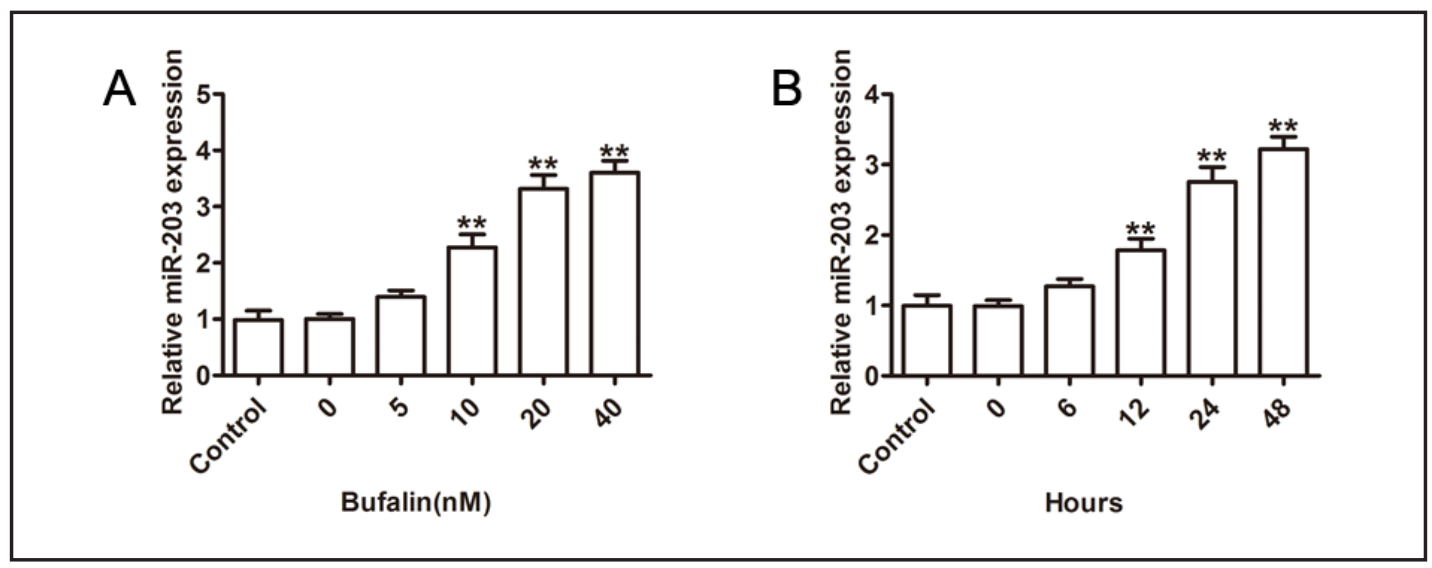

Fig. 3. MiR-203 inhibits the progression of glioma cells. (A) Overexpression of miR-203 significantly suppressed cell proliferation and (C) tumor spheres (D) of U251 and U87 cells. (B) Overexpression of miR203 markedly induced cell apoptosis. (C and D) Overexpression of miR-203 significantly reduced colony formation and the number of neurospheres formed in U251 and U87 cells. (E) Expression of OCT4 and SOX2 in U251 and U87 cells transfected with miR-203 mimics were examined by Western blotting. Expression of miR-27a and miR-34a in CRC cells treated with curcumin and/or AKBA. MiRNA expression was normalized to RNU6B. (B) Western blots of downstream targets of miRNAs. All CRC cell lines were treated with $10 \mu \mathrm{M}$ curcumin and/or $30 \mu \mathrm{M}$ AKBA for $24 \mathrm{~h}$. Cells were lysed, and Western blot analyses were performed on putative downstream targets of miR-34a and miR-27a. $\beta$-actin was used as a loading control. $* \mathrm{P}<0.05$, ** $\mathrm{P}<0.01$ and ${ }^{* * *} \mathrm{P}<0.001$ compared to the corresponding control.

and neurosphere formation were both significantly increased in the anti-miR-203 group compared to the SCR group $(P<0.05)$ (Fig. 4C, 4D). Moreover, OCT4 and SOX2 were both upregulated in the anti-miR-203 group (Fig. 4E).

\section{SPARC is a miR-203 target in glioma cells}

To test whether miR-203 binds the 3'-UTR of SPARC, two pairs of oligonucleotides containing the wild-type or mutated binding site of miR-203 on the SPARC 3'-UTR were synthesized and cloned into a luciferase reporter plasmid vector (Fig. 5A). The vectors miR-203, miR-NR, SPARC-UTR-WT, and SPARC-UTR-MUT were co-transfected into separate cells. Overexpression of miR-203 significantly reduced the luciferase activity of SPARCWT, whereas luciferase activity was unaffected by miR-NR, which contained a mutated miR-203 seed binding site. Meanwhile, miR-203-mediated repression of luciferase activity was reversed when a mutated putative binding site (SPARC-UTR-MUT) was used (Fig. 
Fig. 4. Inhibition of miR203 reverses the effects of bufalin on glioma cells. (A-D) The effect of knockdown of miR-203 on cell proliferation $(\mathrm{A})$, apoptosis (B), colony formation (C), and tumor spheres (D) of U251 and U87 cells in response to bufalin treatment. (E) The expression levels of the OCT4 and SOX2 proteins were evaluated in U251 and U87 cells transfected with miR-203 inhibitors after treatment with bufalin by Western blot analysis.

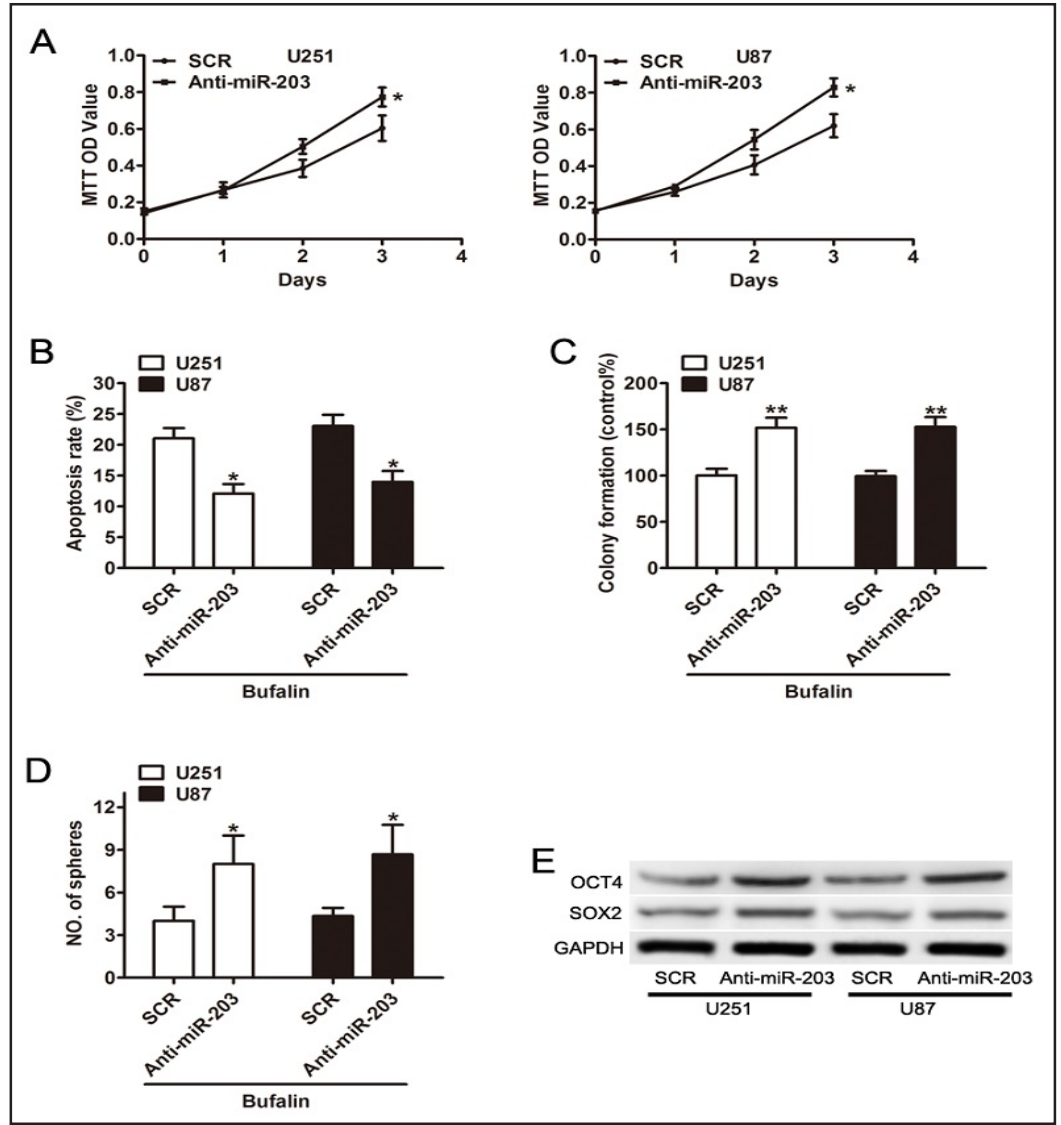

5B). Furthermore, luciferase activity assays were also performed on cells transfected with SPARC-UTR-WT and SPARC-UTR-MUT following treatment with bufalin. Similarly, luciferase activity was significantly reduced in the SPARC-UTR-WT-transfected cells following bufalin treatment, whereas luciferase activity was unaffected in SPARC-UTR-MUT-transfected cells, despite bufalin treatment (Fig. 5C). When U251 and U87 cells were transfected with miR-203, relative SPARC mRNA and protein levels were found to be significantly decreased $(P<0.05)$ (Fig. 5D, 5F). Furthermore, after bufalin treatment, relative SPARC mRNA and protein levels were also significantly decreased $(P<0.05)$ (Fig. 5E, 5G). Taken together, these results suggest that SPARC is a target of miR-203, and bufalin can inhibit SPARC expression via miR-203.

\section{Discussion}

Gliomas are one of the most lethal tumors, with their poor prognosis stemming from their unresponsiveness to conventional cancer therapies, including surgery, radiotherapy, photodynamic therapy, and chemotherapy. Due to the high incidence and mortality of gliomas, development of new therapeutic strategies is urgently needed.

The Chinese traditional medicine ChanSu has been used as an anti-cancer agent for many years. As one of the main components of ChanSu, bufalin has been shown to be an effective anticancer agent in multiple cancer types $[6,9,10,20]$. Bufalin exerts anticancer effects primarily by inhibiting tumor cell proliferation, inhibiting migration, inducing tumor cell apoptosis, and inducing differentiation [21]. One study reported that bufalin inhibits the differentiation and proliferation of CSCs derived from primary osteoscarcoma cells through miR-148a [22]; thus, microRNA expression may be affected by bufalin and may control the mechanism of action in tumors. However, the effect of bufalin on glioma cells and the underlying molecular mechanism of such an effect has yet to be studied. 
Liu et al.: Bufalin Inhibits Cellular Proliferation via MiR-203

Fig. 5. MiR-203 mediated SPARC expression via direct binding to its 3'-UTR. (A) Sequence alignment of has-miR-203 seed sequence with the 3'-UTR of SPARC and its mutated sequence at the complementary sites. (B) Luciferase assay was performed to detect luciferase activity in HEK-293T cells transfected with miR-203 mimics and wild-type or mutated 3'-UTR SPARC. (C) Luciferase assay was performed to detect luciferase activity in U251 cells treated with bufalin and transfected with wild-type or mutated 3'-UTR SPARC. (D) The mRNA levels of SPARC in U251 and U87 cells transfected with miR-203. (E) The mRNA levels of SPARC in U251 and U87 cells treated with bufalin. (F) The expression of SPARC protein in U251 and U87 cells transfected with miR-203. (G) The expression of SPARC protein in U251 and U87 cells treated with bufalin.

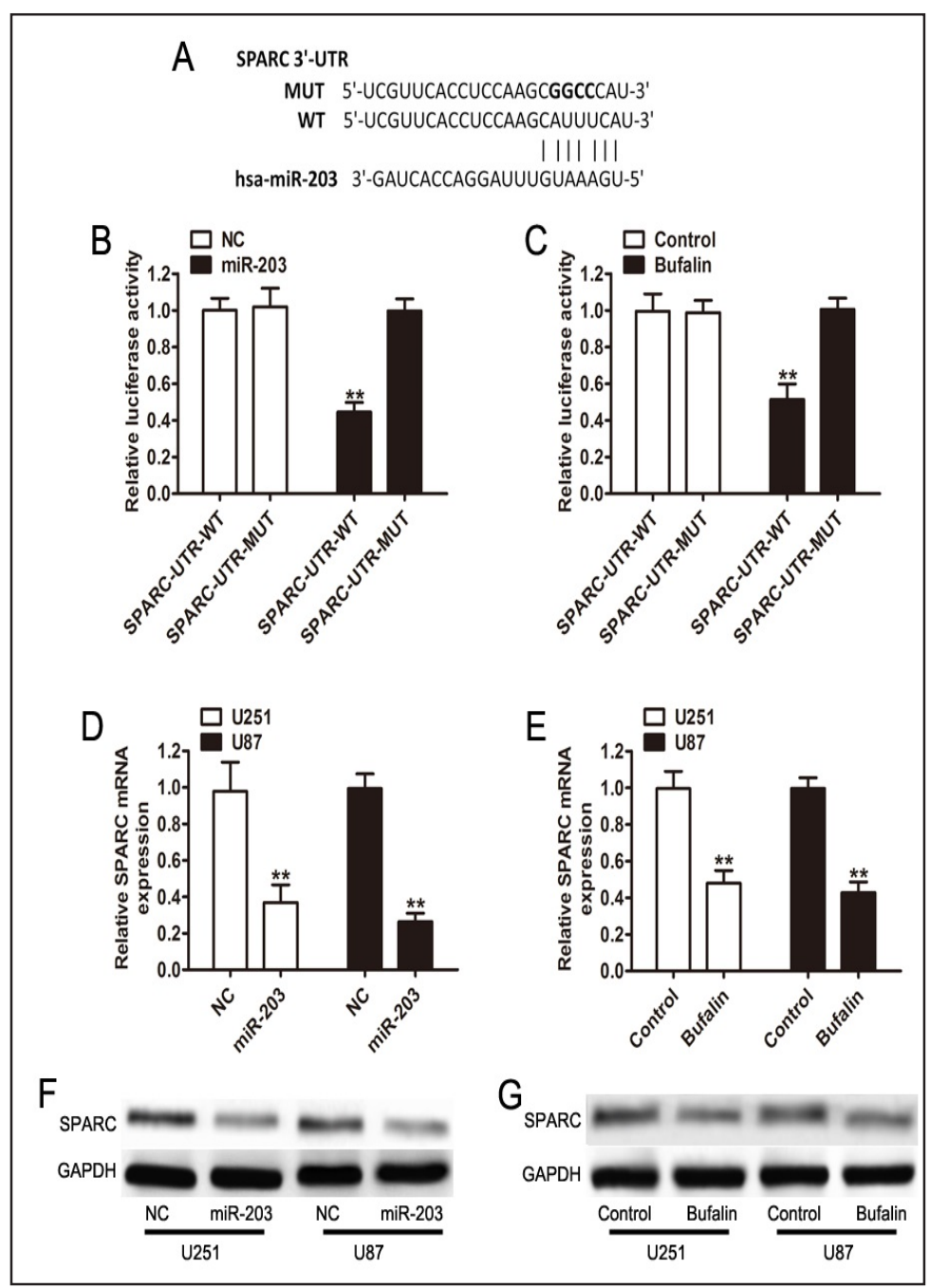

In the present study, we found that bufalin exhibits strong tumor suppressive properties, including the suppression of glioma cell proliferation and the development of stem cell-like phenotypes. Here, we also report the biological role of miR-203 in human glioma. Moreover, we observed that bufalin can induce miR-203 expression in glioma. MiR-203 itself can suppress cell proliferation through the promotion of apoptosis and through blocking the development of stem cell-like phenotypes as shown by a reduction in expression of stem cell markers. However, inhibition of miR-203 can reverse bufalin-mediated effects on cell proliferation. Therefore, bufalin's tumor suppressive abilities may be through the induction of miR-203 expression in glioma cells. As such, miR-203 is an attractive therapeutic agent.

MiR-203 is a tumor-suppressive miRNA that may play a role in various malignancies, including prostate cancer, pancreatic cancer, esophageal cancer and gliomas [15, 23-25]. MiR-203 expression levels were reported to be significantly lower in glioma tissue, and the levels negatively and positively correlated with WHO grade and survival, respectively $[17,24,26,27]$. MiR-203 has several possible protein targets that are oncogenic in nature. One potential target is the secreted protein acidic and rich in cysteine (SPARC) gene. One previous study reported that miR-203 regulates cell differentiation to inhibit lung metastasis by suppressing SPARC, which is involved in prometastatic activity via extracellular matrix remodeling [24]. SPARC is a matricellular protein that is expressed intracellularly and is secreted into the extracellular matrix. Prior studies have shown that SPARC promotes cancer cell migration and invasion and correlates with poor overall survival in carcinomas [28]. 
SPARC is oncogenic in nature and highly amplified in human glioma. SPARC expression is therefore essential for glioma development and proliferation [29].

In our study, we demonstrated that miR-203 can target SPARC. Overexpression of miR-203 can significantly suppress SPARC expression. Our data also showed that bufalin treatment can reduce SPARC expression in glioma. These results provide a possible clue regarding the role of miR-203 as a tumor suppressor in glioma and its possible interplay with SPARC expression. In addition, we also hypothesized that bufalin's promising effects on glioma are mediated through miR-203 and SPARC.

Furthermore, recent preclinical and clinical data identified a potential anti-cancer effect of $\mathrm{Na}+/ \mathrm{K}+$ ATPase inhibition in various indications [30,31], and bufalin is predominantly a $\mathrm{Na}+/ \mathrm{K}+\mathrm{ATP}$ enzyme inhibitor [32]. As with other cardiac glycoside drugs, the anticancer effects of bufalin are exerted primarily by inhibiting $\mathrm{Na}+\mathrm{K}+$ ATPase [31]. Therefore, to provide a mechanistic approach of the observed effects, further research should be performed to identify the possible cross-talk of this natural cardiotonic steroid with $\mathrm{Na}+\mathrm{K}+$ ATPase.

\section{Conclusion}

Our study suggests that bufalin's therapeutic effects occur via the induction of miR-203 expression, which negatively regulates SPARC expression in glioma.

\section{Acknowledgements}

The present study was supported in part by grants from the Key Science and Technology Project of Hainan Province (ZDXM2015070), the Natural Science Foundation of China (81460261, 81760308).

\section{Disclosure Statement}

There are no competing interests.

\section{References}

1 Bondy ML, Scheurer ME, Malmer B, Barnholtz-Sloan JS, Davis FG, Il'yasova D, Kruchko C, McCarthy BJ, Rajaraman P, Schwartzbaum JA, Sadetzki S, Schlehofer B, Tihan T, Wiemels JL, Wrensch M, Buffler PA: Brain Tumor Epidemiology C: Brain tumor epidemiology: consensus from the Brain Tumor Epidemiology Consortium. Cancer 2008;113:1953-1968.

-2 Bao S, Wu Q, McLendon RE, Hao Y, Shi Q Hjelmeland AB, Dewhirst MW, Bigner DD, Rich JN: Glioma stem cells promote radioresistance by preferential activation of the DNA damage response. Nature 2006;444:756-760.

-3 Slingerland M, Cerella C, Guchelaar HJ, Diederich M, Gelderblom H: Cardiac glycosides in cancer therapy: from preclinical investigations towards clinical trials. Invest New Drugs 2013;31:1087-1094.

-4 Steyn PS, van Heerden FR: Bufadienolides of plant and animal origin. Nat Prod Rep 1998;15:397-413.

5 Alevizopoulos K, Calogeropoulou T, Lang F, Stournaras C: Na+/K+ ATPase inhibitors in cancer. Curr Drug Targets 2014;15:988-1000.

6 Zhu Z, Sun H, Ma G, Wang Z, Li E, Liu Y, Liu Y: Bufalin induces lung cancer cell apoptosis via the inhibition of PI3K/Akt pathway. Int J Mol Sci 2012;13:2025-2035.

7 Wang Y, Lonard DM, Yu Y, Chow DC, Palzkill TG, Wang J, Qi R, Matzuk AJ, Song X, Madoux F, Hodder P, Chase P, Griffin PR, Zhou S, Liao L, Xu J, O'Malley BW: Bufalin is a potent small-molecule inhibitor of the steroid receptor coactivators SRC-3 and SRC-1. Cancer Res 2014;74:1506-1517. 


\section{Cellular Physiology Cell Physiol Biochem 2017;44:671-681 \begin{tabular}{ll|l} 
DOI: 10.1159/000485279 & O 2017 The Author(s). Published by S. Karger AG, Basel \\
wwww.kargercom/cpb
\end{tabular}

Liu et al.: Bufalin Inhibits Cellular Proliferation via MiR-203

8 Meng Z, Yang P, Shen Y, Bei W, Zhang Y, Ge Y, Newman RA, Cohen L, Liu L, Thornton B, Chang DZ, Liao Z, Kurzrock R: Pilot study of huachansu in patients with hepatocellular carcinoma, nonsmall-cell lung cancer, or pancreatic cancer. Cancer 2009;115:5309-5318.

-9 Takai N, Ueda T, Nishida M, Nasu K, Narahara H: Bufalin induces growth inhibition, cell cycle arrest and apoptosis in human endometrial and ovarian cancer cells. Int J Mol Med 2008;21:637-643.

10 Zhu Z, Li E, Liu Y, Gao Y, Sun H, Wang Y, Wang Z, Liu X, Wang Q, Liu Y: Bufalin induces the apoptosis of acute promyelocytic leukemia cells via the downregulation of survivin expression. Acta Haematol 2012;128:144150.

11 Bartel DP: MicroRNAs: target recognition and regulatory functions. Cell 2009;136:215-233.

$\checkmark 12$ Chen PH, Cheng CH, Shih CM, Ho KH, Lin CW, Lee CC, Liu AJ, Chang CK, Chen KC: The Inhibition of microRNA-128 on IGF-1-Activating mTOR Signaling Involves in Temozolomide-Induced Glioma Cell Apoptotic Death. PLoS One 2016;11:e0167096.

13 Moller HG, Rasmussen AP, Andersen HH, Johnsen KB, Henriksen M, Duroux M: A systematic review of microRNA in glioblastoma multiforme: micro-modulators in the mesenchymal mode of migration and invasion. Mol Neurobiol 2013;47:131-144.

14 Qi Z, Cai S, Cai J, Chen L, Yao Y, Chen L, Mao Y: miR-491 regulates glioma cells proliferation by targeting TRIM28 in vitro. BMC Neurol 2016;16:248.

15 He J, Deng Y, Yang G, Xie W: MicroRNA-203 down-regulation is associated with unfavorable prognosis in human glioma. J Surg Oncol 2013;108:121-125.

16 Liao H, Bai Y, Qiu S, Zheng L, Huang L, Liu T, Wang X, Liu Y, Xu N, Yan X, Guo H: MiR-203 downregulation is responsible for chemoresistance in human glioblastoma by promoting epithelial-mesenchymal transition via SNAI2. Oncotarget 2015;6:8914-8928.

17 Chen Z, Li D, Cheng Q Ma Z, Jiang B, Peng R, Chen R, Cao Y, Wan X: MicroRNA-203 inhibits the proliferation and invasion of U251 glioblastoma cells by directly targeting PLD2. Mol Med Rep 2014;9:503-508.

18 Noguchi S, Mori T, Otsuka Y, Yamada N, Yasui Y, Iwasaki J, Kumazaki M, Maruo K, Akao Y: Anti-oncogenic microRNA-203 induces senescence by targeting E2F3 protein in human melanoma cells. J Biol Chem 2012;287:11769-11777.

19 Pal D, Mukhopadhyay D, Ramaiah MJ, Sarma P, Bhadra U, Bhadra MP: Regulation of Cell Proliferation and Migration by miR-203 via GAS41/miR-10b Axis in Human Glioblastoma Cells. PloS One 2016;11:e0159092.

20 Zhang N, Xie Y, Tai Y, Gao Y, Guo W, Yu W, Li J, Feng X, Hao J, Zhao X, Liao Y, Jiang W, Liu G, Deng W, Cui X: Bufalin Inhibits hTERT Expression and Colorectal Cancer Cell Growth by Targeting CPSF4. Cell Physiol Biochem 2016;40:1559-1569.

21 Shen S, Zhang Y, Wang Z, Liu R, Gong X: Bufalin induces the interplay between apoptosis and autophagy in glioma cells through endoplasmic reticulum stress. Int J Biol Sci 2014;10:212-224.

22 Chang Y, Zhao Y, Gu W, Cao Y, Wang S, Pang J, Shi Y: Bufalin Inhibits the Differentiation and Proliferation of Cancer Stem Cells Derived from Primary Osteosarcoma Cells through Mir-148a. Cell Physiol Biochem 2015;36:1186-1196.

23 Saini S, Majid S, Yamamura S, Tabatabai L, Suh SO, Shahryari V, Chen Y, Deng G, Tanaka Y, Dahiya R: Regulatory Role of mir-203 in Prostate Cancer Progression and Metastasis. Clin Cancer Res 2011;17:52875298.

-24 Benaich N, Woodhouse S, Goldie SJ, Mishra A, Quist SR, Watt FM: Rewiring of an epithelial differentiation factor, miR-203, to inhibit human squamous cell carcinoma metastasis. Cell Rep 2014;9:104-117.

-25 Ren ZG, Dong SX, Han P, Qi J: miR-203 promotes proliferation, migration and invasion by degrading SIK1 in pancreatic cancer. Oncol Rep 2016;35:1365-1374.

26 He JH, Li YM, Li YG, Xie XY, Wang L, Chun SY, Cheng WJ: hsa-miR-203 enhances the sensitivity of leukemia cells to arsenic trioxide. Exp Ther Med 2013;5:1315-1321.

27 Dontula R, Dinasarapu A, Chetty C, Pannuru P, Herbert E, Ozer H, Lakka SS: MicroRNA 203 Modulates Glioma Cell Migration via Robo1/ERK/MMP-9 Signaling. Genes Cancer 2013;4:285-296.

28 Chin D, Boyle GM, Williams RM, Ferguson K, Pandeya N, Pedley J, Campbell CM, Theile DR, Parsons PG, Coman WB: Novel markers for poor prognosis in head and neck cancer. Int J Cancer 2005;113:789-797.

29 Schultz CR, Golembieski WA, King DA, Brown SL, Brodie C, Rempel SA: Inhibition of HSP27 alone or in combination with pAKT inhibition as therapeutic approaches to target SPARC-induced glioma cell survival. Mol Cancer 2012;11:20. 
Liu et al.: Bufalin Inhibits Cellular Proliferation via MiR-203

30 Lang F, Stournaras C: Ion channels in cancer: future perspectives and clinical potential. Philos Trans R Soc Lond B Biol Sci 2014;369:20130108.

-31 Dimas K, Papadopoulou N, Baskakis C, Prousis KC, Tsakos M, Alkahtani S, Honisch S, Lang F, Calogeropoulou T, Alevizopoulos K, Stournaras C: Steroidal cardiac Na+/K+ ATPase inhibitors exhibit strong anti-cancer potential in vitro and in prostate and lung cancer xenografts in vivo. Anticancer Agents Med Chem 2014;14:762-770.

-32 Alevizopoulos K, Dimas K, Papadopoulou N, Schmidt EM, Tsapara A, Alkahtani S, Honisch S, Prousis KC, Alarifi S, Calogeropoulou T, Lang F, Stournaras C: Functional characterization and anti-cancer action of the clinical phase II cardiac $\mathrm{Na}+/ \mathrm{K}+$ ATPase inhibitor istaroxime: in vitro and in vivo properties and cross talk with the membrane androgen receptor. Oncotarget 2016;7:24415-24428. 